Article

\title{
Combined Effect of Nitrofurantoin and Plant Surfactant on Bacteria Phospholipid Membrane
}

\author{
Monika Rojewska, Wojciech Smułek *(D), Krystyna Prochaska and Ewa Kaczorek (1) \\ Institute of Chemical Technology and Engineering, Poznan University of Technology, 60-695 Poznan, Poland; \\ monika.rojewska@put.poznan.pl (M.R.); Krystyna.Prochaska@put.poznan.pl (K.P.); \\ ewa.kaczorek@put.poznan.pl (E.K.) \\ * Correspondence: wojciech.smulek@put.poznan.pl; Tel.: +48-61-6653671 \\ Academic Editor: Ricardo L. Mancera

Received: 27 April 2020; Accepted: 28 May 2020; Published: 28 May 2020

\begin{abstract}
Due to the increasing use of antibiotics, measures are being taken to improve their removal from the natural environment. The support of biodegradation with natural surfactants that increase the bioavailability of impurities for microorganisms that degrade them, raises questions about their effect on bacterial cells. In this paper we present analysis of the interaction of nitrofurantoin (NFT) and saponins from the Saponaria officinalis on the environmental bacteria membrane and the model phospholipid membrane mimicking it. A wide perspective of the process is provided with the Langmuir monolayer technique and membrane permeability test with bacteria. The obtained results showed that above critical micelle concentration $(\mathrm{CMC})$, saponin molecules are incorporated into the POPE monolayer, but the NFT impact was ambiguous. What is more, differences in membrane permeability between the cells exposed to NFT in comparison to that of the non-exposed cells were observed above 1.0 CMC for Achromobacter sp. KW1 or above 0.5 CMC for Pseudomonas sp. MChB. In both cases, NFT presence lowered the membrane permeability. Moreover, the Congo red adhesion to the cell membrane also decreased in the presence of a high concentration of surfactants and NFT. The results suggest that saponins are incorporated into the bacteria membrane, but their sugar hydrophilic part remains outside, which modifies the adsorption properties of the cell surface as well as the membrane permeability.
\end{abstract}

Keywords: antibiotics; bacteria; membrane permeability; nitrofurantoin; phospholipids membrane; POPE; saponins; surfactants

\section{Introduction}

Excessive use of antibiotics and the lack of their control when released into the environment have contributed to deterioration of the ecosystem. Many antibiotics can get into the natural environment due to the fact that modern water treatment plants have not been designed to completely remove these substances. In consequence, antibiotics can enter the environment and accumulate in ecosystems. The presence of antibiotics in the environment can be harmful to many useful microbial communities as a consequence of their bacteriocidic and bacteriostatic actions. This problem also refers to nitrofuran derivatives, which are administered to humans and animals. Their representative derivative is nitrofurantoin (NFT), which is a broad spectrum antibiotic used to treat bladder infections of humans as well as in veterinary medicine [1,2]. NFT is only partially metabolized by the mammal organisms, while the majority of the compound is excreted unchanged and goes to the sewage and to the environment. For this reason, new solutions that would allow removal of the pharmaceutical product from the ecosystem are searched for. One of the promising methods is the biodegradation of the antibiotic in the natural environment using bacteria and saponins. Pacholak et al. [3] have shown that some bacterial strains use nitrofurantoin as a source of carbon and energy. Nitrofurantoin removal 
by selected microbial cultures ranged from $50 \%$ to $90 \%$ in 28 days, depending on the bacterial strain. On the other hand, this biodegradation process is quite long and relatively low effective. However, surfactants are expected to support the process to obtain its better efficiency. The presence of a surfactant promotes, among others, an increase in the permeability of biological membranes. To avoid additional contamination of the environment by artificial surfactants, the natural ones, like saponins, would be especially useful [4]. Saponins found application in enhancing biodegradation of persistent organic pollutants like halogenated phenols [5] or polycyclic aromatic hydrocarbons (PAH's) [6]. Hence, it can be expected that saponins' would promote the increase in bioavailability of the antibiotic for bacteria and as a result accelerate the biodegradation process [7-9]. However, in order to reasonably control the surfactant-enhanced biodegradation process, it is necessary to thoroughly understand the mechanism of nitrofurantoin interaction with the bacterial surface membrane in the presence of saponins at the molecular level. Currently, a lot of research has been made on the potential bioactivity of saponins focused only on the overall cellular response to many stimuli. However, these studies cannot provide clear molecular information on the mechanism of action of saponins on the membrane. The physiological mode of the cells adjustment in response to the external stimuli such as temperature or mechanical force is modification of the lipid composition of their membrane to maintain functional integrity [10]. From this point of view, the Langmuir monolayers technique is an alternative to experiments using living cell because it allows investigation of analogues of biological membranes. It is possible because a biological membrane can be considered as composed of two weakly coupled monolayers. Lipid monolayers formed at the air-water interface are a versatile membrane model for studying the interaction and physicochemical properties of monolayer components. The simplicity of creating model lipid systems allows investigation of the interaction of real biological membranes with bioactive substances such as saponins. Both the composition and molecular density of Langmuir monolayers can be easily controlled. Moreover, these model lipid systems can be easily characterized using a number of surface characterization techniques (surface pressure and surface potential, surface rheology, Brewster microscopy and fluorescence, neutron and X-ray scattering, ellipsometry or reflection spectroscopy (UV and IR) [11].

The Langmuir monolayer technique is also suitable to investigate the interaction between saponin and biological membrane [11,12]. This technique allows the creation of a monolayer imitating biomembrane by spreading organic compounds (e.g., lipids, phospholipids and glycolipids) on the aqueous subphase. In effect, amphiphilic lipids form an insoluble monolayer called the Langmuir film, where the molecules are oriented with their hydrophobic tail towards the gas phase and the hydrophilic head group anchored in the aqueous subphase. The formation of model membrane involves dissolving lipids in an organic solvent (e.g., chloroform) and then this solution is deposited onto the subphase. While the solvent evaporates, the lipid molecules spread to form a Langmuir film at the air/water interface. The measuring principle is based on the fact that the monolayer is compressed continuously by Teflon barriers during the experiment and the change in surface pressure vs. the monomolecular layer area at this time are recorded. The surface pressure is recorded by a Wilhelmy plate connected to a pressure sensor. The Wilhelmy plate is immersed into the subphase and the forces acting on the plate include gravity, surface tension and upward forces. The monolayer compressed to the surface pressure of $30 \mathrm{mN} / \mathrm{m}$ represents the natural biological membrane conditions [13-15]. Moreover, lipids play a very important role in maintaining the stiffness of the membrane and determine its permeability to other substances.

Hence, in this study we are concerned with finding out the ways in which saponins, NFT and their mixtures interact with the phospholipids monolayer. With these ideas in mind, we studied these interactions on a model of cellular membrane in the form of Langmuir monolayer consisting of selected lipid 2-oleoyl-1-palmitoyl-sn-glycero-3-phosphoethanolamine (POPE). The lipid part of the phospholipid represents the major fatty acids found in cell membranes structures of environmental bacteria. Saponins were extracted from Saponaria officinalis roots. When trying to link molecular structure with interfacial behavior it should be kept in mind that the extracts can differ in the saponins 
content and composition. In addition, impurities (residual plant substances) may be present and affect interfacial properties. Saponins are surface-active substances so we expect that they interact with the model membrane, but we wanted to determine in detail their interaction mechanism and establish the influence of natural surfactants concentration on the membrane stability in the nitrofurantoin environment. This knowledge seems to be crucial for the possible use of saponins as substances supporting the process of nitrofurantoin biodegradation.

\section{Results}

\subsection{POPE Membrane Model Tests}

The amphiphilic character of saponins allows them to aggregate in aqueous solutions and interact with membrane components. With this in mind we study how the change in concentration of saponins influence their interaction with the model membrane and consequently disturb the membrane fluidity. In our experiments, after formation of a phospholipid monolayer at the buffer subphase $(\mathrm{pH}=7.4)$ we exchanged the subphase to deliver saponins or/and NTF to the investigated system. The subphase flow was slow enough $(13 \mathrm{~mL} / \mathrm{min})$ to neither disturb the structure of the monolayer nor cause its destruction in the process of replacement of the bulk phase with a new one. The relaxation experiment consisted of keeping the surface pressure $(\pi)$ constant, and recording the area (A) as a function of time.

First, POPE was spread on the buffer subphase $(\mathrm{pH}=7.4)$ to form a monolayer. After $15 \mathrm{~min}$, the film was compressed to the surface pressure of $30 \mathrm{mN} / \mathrm{m}$, and after compression the surface per molecule was estimated as $\mathrm{A}_{0}$. When introducing Saponaria extract into the subphase, a change in surface value per molecule was observed in the monolayer $(A(t))$. If this value $(A(t))$ is greater than the $A_{0}\left(A(t) / A_{0}>1\right)$, an increase in the area per molecule caused by the embedded saponin molecule in the phospholipid monolayer is indicated. Otherwise, if $\mathrm{A}(\mathrm{t}) / \mathrm{A}_{0}<1$, there is a surface area loss in the monolayer, so it can be assumed that the phospholipid molecules desorb from the monolayer and dissolve in the subphase. The $\mathrm{A} / \mathrm{A}_{0}$ parameter was therefore a measure of the monolayer stability.

Two effects can be observed as a result of the interaction of the membrane with the molecules introduced in the subphase. If the particles build into the model monolayer then the surface area between the Teflon barriers will increase to maintain the set surface pressure $(30 \mathrm{mN} / \mathrm{m})$. Otherwise, we can observe a reduction of the surface area between the Teflon barriers as a consequence of desorption of molecules from the monolayer. In this way, the relaxation/penetration experiment provided important information about surface properties and stability of model membrane in the presence of saponins and NTF as a result of their mutual interactions. At first, pure lipid was spread at the water-air interface to obtain the initial surface pressure of approximately $30 \mathrm{mN} / \mathrm{m}$. Then $150 \mathrm{~mL}$ of the saponins solution in various concentrations were injected into the water subphase, beneath the lipid film, to reach a final concentration of $0.5 \mathrm{~g} / \mathrm{L}(0.5 \mathrm{CMC}$ - critical micellar concentration), $1 \mathrm{~g} / \mathrm{L}$ (1.0 CMC) and $1.5 \mathrm{~g} / \mathrm{L}(1.5 \mathrm{CMC})$, respectively. In aqueous solutions at low surfactant concentrations, surfactant molecules appeared in the form of monomers. However, above a certain critical concentration called critical micellar concentration (CMC), surfactant molecules can exist as both monomers and micellar associates. On the basis of the surface tension isotherm, the CMC value of analyzed Saponaria extract was estimated about $1 \mathrm{~g} / \mathrm{L}$. In our experiments we chose such concentrations of saponins to permit investigation of the effect of their aggregation process on the adsorption. To avoid aggregation of saponins in the subphase, a solution of $0.5 \mathrm{~g} / \mathrm{L}$ was prepared, corresponding to a saponins concentration below the CMC. The results are presented in Figure 1. The saponins penetrate from the subphase into the condensed lipid monolayer and interrupt its structure, because the $\mathrm{A} / \mathrm{A}_{0}$ value increased in time.

According to the results, the injection of saponins changed drastically the monolayer relaxation behavior as the saponins penetrated from the subphase into the condensed lipid monolayers. The higher the saponins concentration in the subphase, the faster the incorporation of saponins into the monolayer was observed. When the plant extract was used at $0.5 \mathrm{CMC}$, the increase in the initial surface area 
value $\left(\mathrm{A}_{0}\right)$ was doubled after about $1700 \mathrm{~s}$, whereas for the test with Saponaria extract at $1.5 \mathrm{~g} / \mathrm{L}$ (i.e., $1.5 \mathrm{CMC})$, the same effect was reached after about $1200 \mathrm{~s}$.

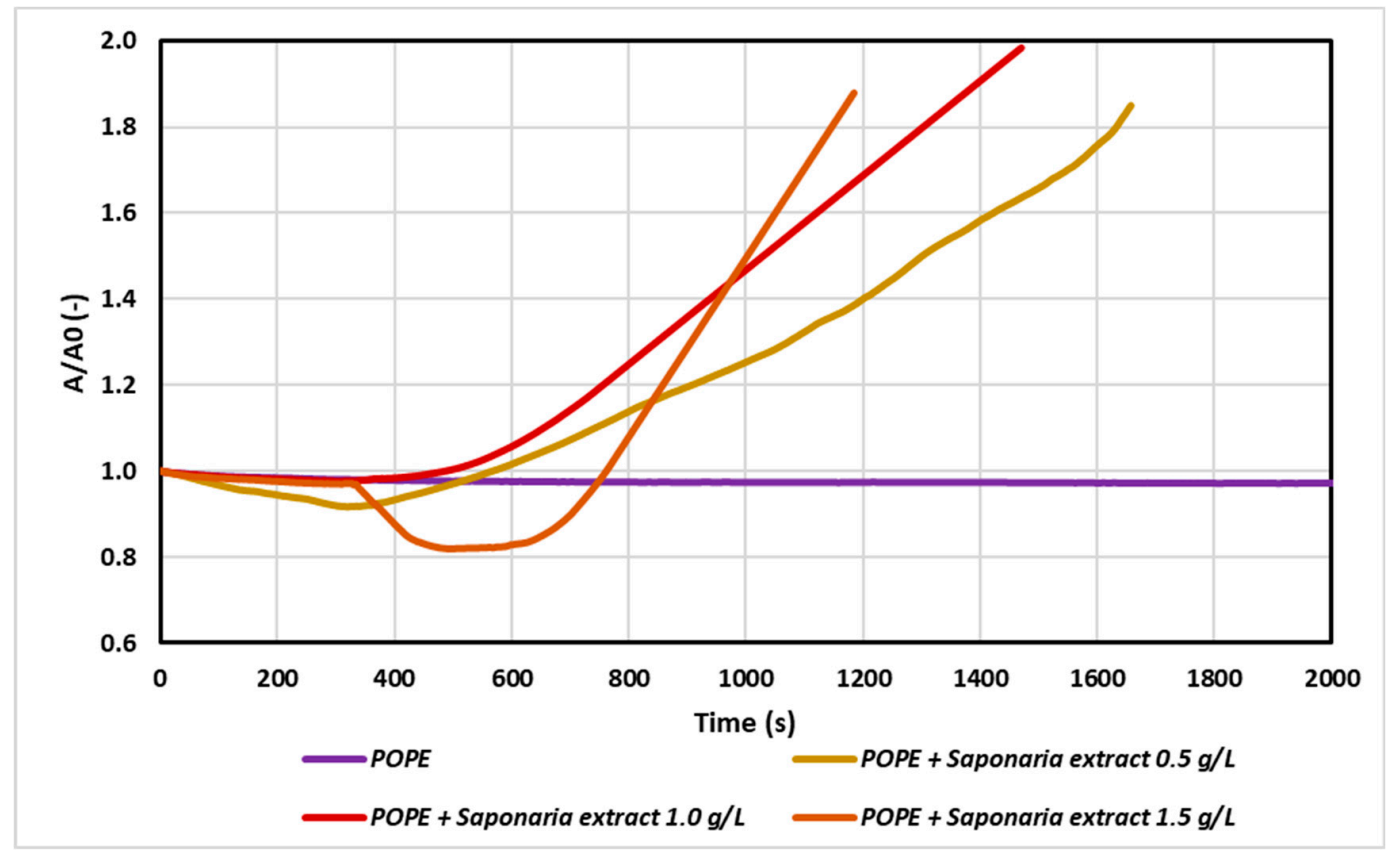

Figure 1. Relative area-time curves for the POPE monolayer in the control sample (with buffer only) and with saponins injected into the subphase in concentrations: $0.5 \mathrm{~g} / \mathrm{L} ; 1.0 \mathrm{~g} / \mathrm{L}$ and $1.5 \mathrm{~g} / \mathrm{L}$ (i.e., 0.5 of critical micellar concentration-CMC; 1.0 CMC and 1.5 CMC, respectively). The plot shows the normalized area per molecule $\left(\mathrm{A} / \mathrm{A}_{0}\right)$ as a function of time, where $\mathrm{A}_{0}=\mathrm{A}(\mathrm{t}=0)$.

In the next stage of our study, the effect of nitrofurantoin (NFT) concentration on the POPE monolayer was analyzed. The addition of NFT to the subphase slightly influenced the relaxation of POPE monolayers, even if the amount of antibiotic was increased significantly. At higher NFT concentrations a decrease in the $\mathrm{A} / \mathrm{A}_{0}$ value over time was observed.

A more visible impact of NFT concentration was observed only for the systems containing saponins in the subphase (Figure 2). In the system with NFT concentration sixfold higher than that used in the previous experiment, a decrease in the value of $\mathrm{A} / \mathrm{A}_{0}$ was observed after about $1000 \mathrm{~s}$. Thus, a high concentration of the antibiotic in the model phospholipid membrane environment is not conducive to the process of penetration of saponins through the monolayer, especially during prolonged exposure of the membrane to these compounds. It can be assumed that there are interactions between saponins and nitrofurantoin, which begin to compete with the interactions of saponins with a phospholipid monolayer and as a result decelerate penetration process of saponins by POPE.

At the next stage of the study, we studied the systems of a constant concentration of nitrofurantoin $(5 \mathrm{mg} / \mathrm{L})$ and the concentrations of saponins corresponding to $0.5,1.0$ and $1.5 \mathrm{CMC}$ in the subphase. The results are presented in Figure 3.

The faster penetration of natural surfactant molecules into the monolayer with increasing saponins concentration was observed for both the samples with Saponaria extract as well as those with combined NFT and Saponaria extract injected. On the other hand, the character of the relaxation curves recorded for the systems with or without addition of NFT in the subphase was different from that of the above curves. However, the impact of the NFT addition on the saponins containing system is ambiguous. At the concentration of the plant surfactant corresponding to $0.5 \mathrm{CMC}$ the surfactants incorporation into the phospholipid monolayer was found to decrease after $500 \mathrm{~s}$. For the same system, the increase in the surface area $\mathrm{A}$ in the relation to the initial surface area $\mathrm{A}_{0}$ reached about $30 \%$ after $1500 \mathrm{~s}$, whereas for the system without the addition of NFT the observed increase in area was larger and equal to about 
$70 \%$. The opposite effect was obtained in the experiments with NFT and the Saponaria extract, in which the concentration of saponins was higher (1.0 CMC or 1.5 CMC). In this case, the addition of NFT to the systems containing saponins made the surfactant molecules to faster penetrate the phospholipid monolayer, especially at the beginning of the relaxation process.

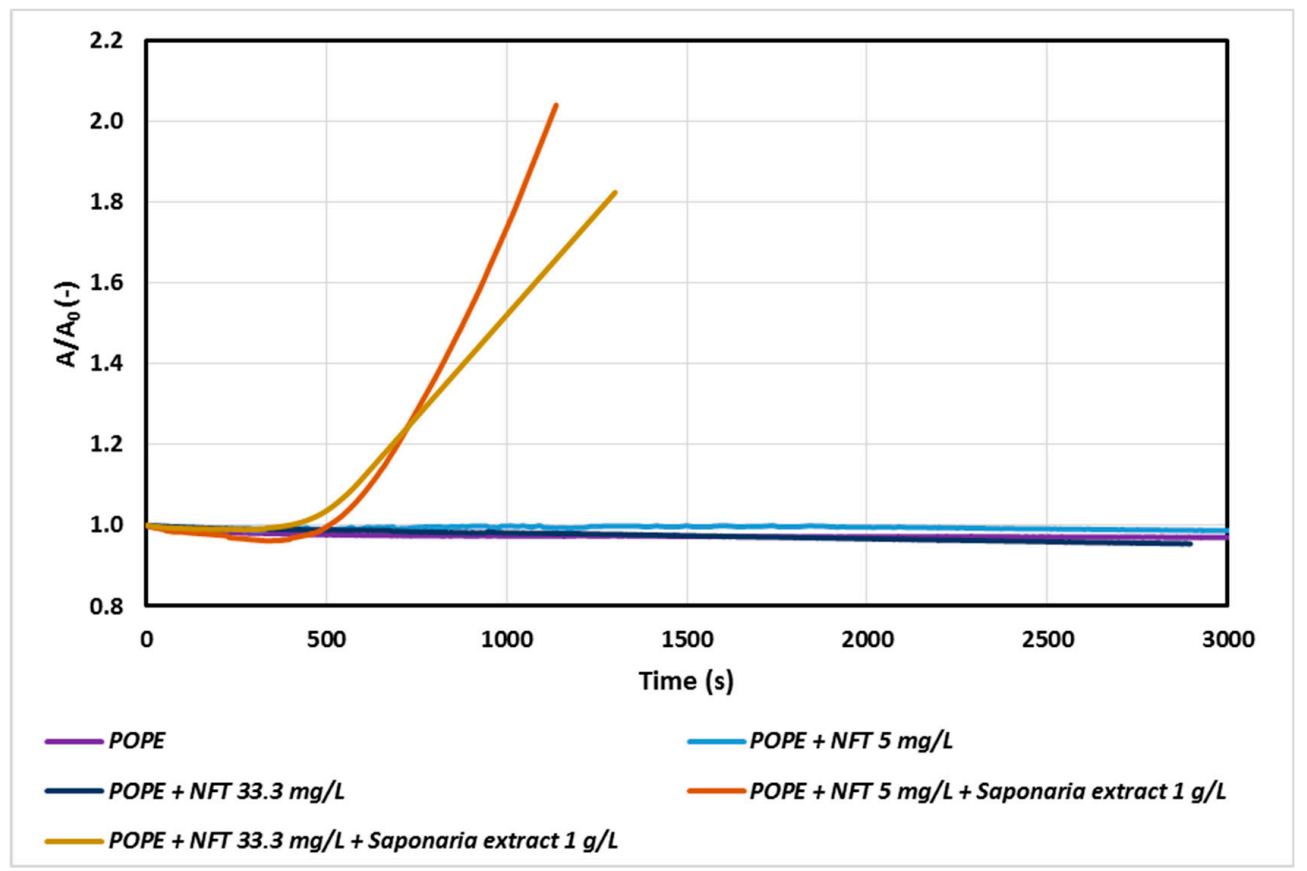

Figure 2. Relative area-time curves for POPE monolayer with NFT or with NFT and extract concentrations $1.0 \mathrm{~g} / \mathrm{L}$ saponins injected into the subphase.

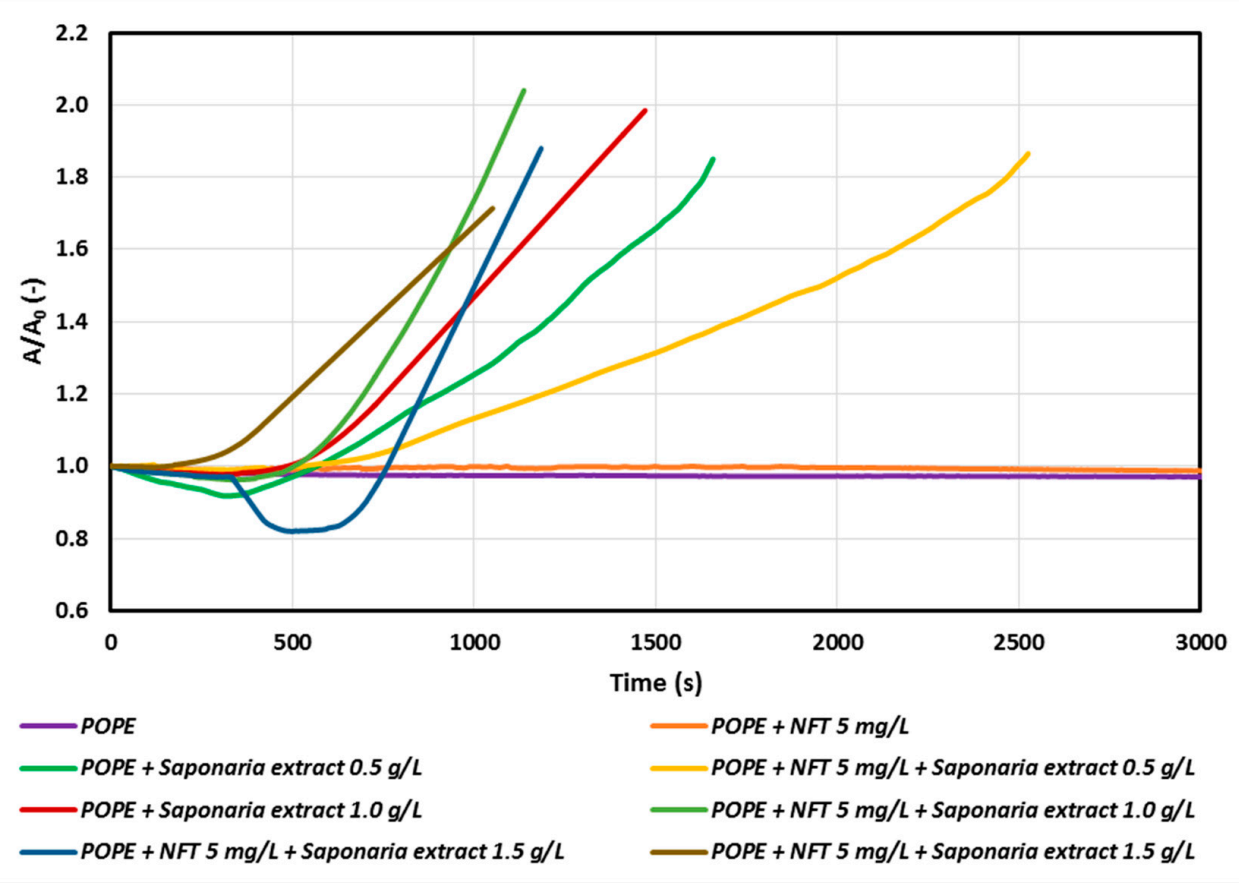

Figure 3. Relative area-time curves for the POPE monolayer with $5 \mathrm{mg} / \mathrm{L}$ of NFT and saponins-rich extract injected into the subphase. 


\subsection{Bacteria Membrane Permeability}

Further experiments focused on the measurements of membrane permeability in biological systems using a crystal violet assay. The two strains, Pseudomonas sp. MChB and Achromobacter sp. KW1 of high content of fatty acids present also in POPE, were chosen for the tests. The results are presented in Figure 4. For the Pseudomonas sp. MChB control samples, i.e., those non-exposed to NFT or plant extract, the permeability was relatively high and exceeded $90 \%$. Moreover, no statistically important changes in the cell membrane permeability were observed up to the critical micelle concentration equal to 1 . The same observations were made after $15 \mathrm{~min}$ (Figure 4a) and $120 \mathrm{~min}$ (Figure $4 \mathrm{~b}$ ). At the plant surfactant concentrations above $1 \mathrm{CMC}$ the permeability decreased significantly, which was even more noticeable after $120 \mathrm{~min}$ than after $15 \mathrm{~min}$. After $120 \mathrm{~min}$ and at $1.5 \mathrm{CMC}$, the cells membrane permeability was equal to $65 \%$. What is worth mentioning there were no differences between the cells exposed to NFT and those non-exposed to NFT below CMC. When the extract concentration exceeded CMC, the addition of NFT led to a slight decrease in the membrane permeability.

The results obtained for Achromobacter sp. KW1 were relatively similar, however some important differences should be indicated. The strain cells were characterized by lower membrane permeability than those of Pseudomonas sp. MChB, which in control samples reached ca. 40\% (Figure 4c). With increasing Saponaria extract concentration the permeability rose up to $50 \%$ at $0.1 \mathrm{CMC}$ after $15 \mathrm{~min}$ and at $0.5 \mathrm{CMC}$ after $120 \mathrm{~min}$. What is more, the further increase in the plant surfactant concentration led to a decrease in the cell membrane permeability, like it was observed for Pseudomonas sp. MChB cells. Moreover, at the Saponaria extract concentration higher than $0.1 \mathrm{CMC}$, the Achromobacter sp. KW1 cells exposed to NFT showed lower permeability than the cells in the samples with surfactants only.
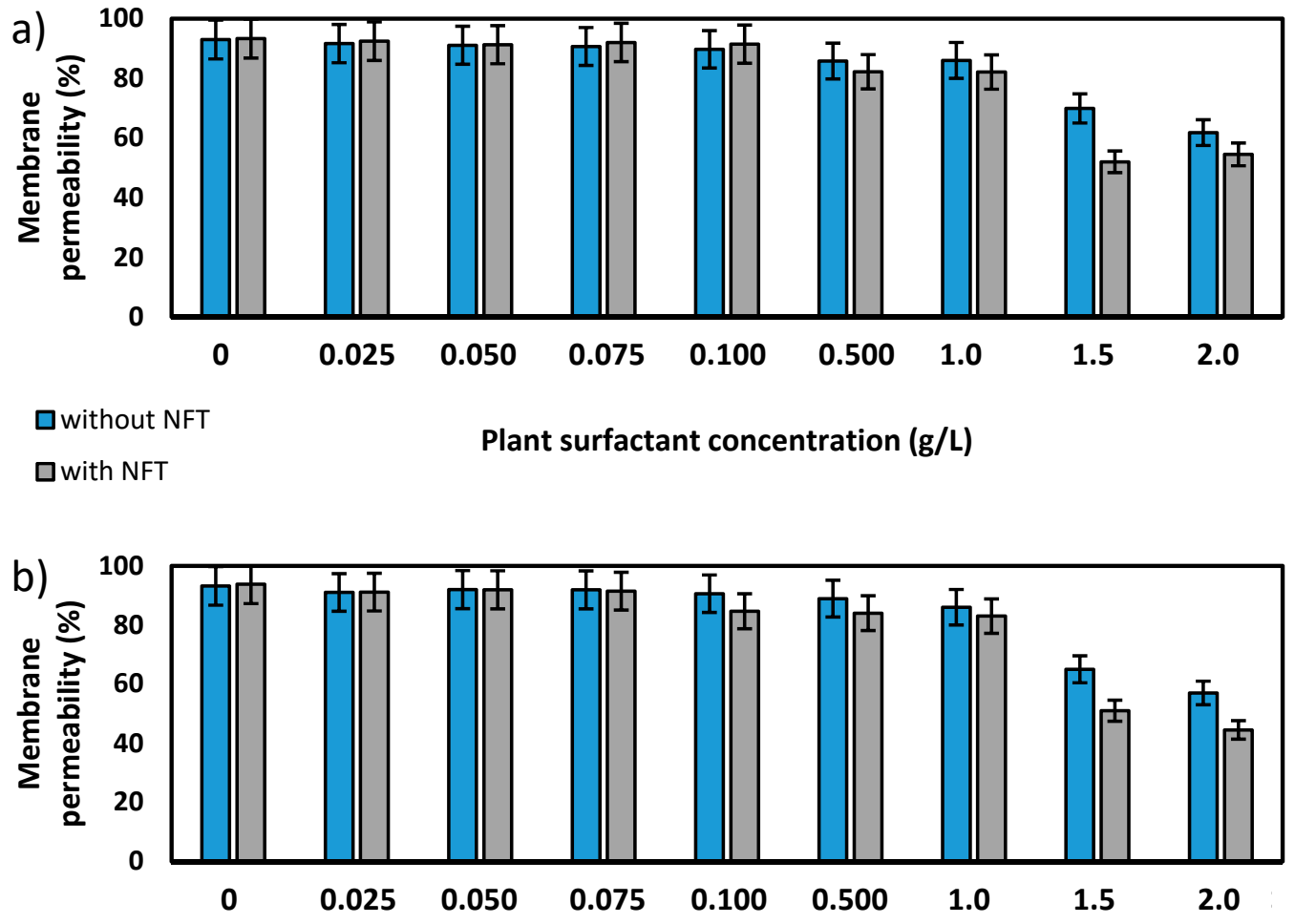

$\square$ without NFT

$\square$ with NFT

Plant surfactant concentration $(\mathrm{g} / \mathrm{L})$

Figure 4. Cont. 

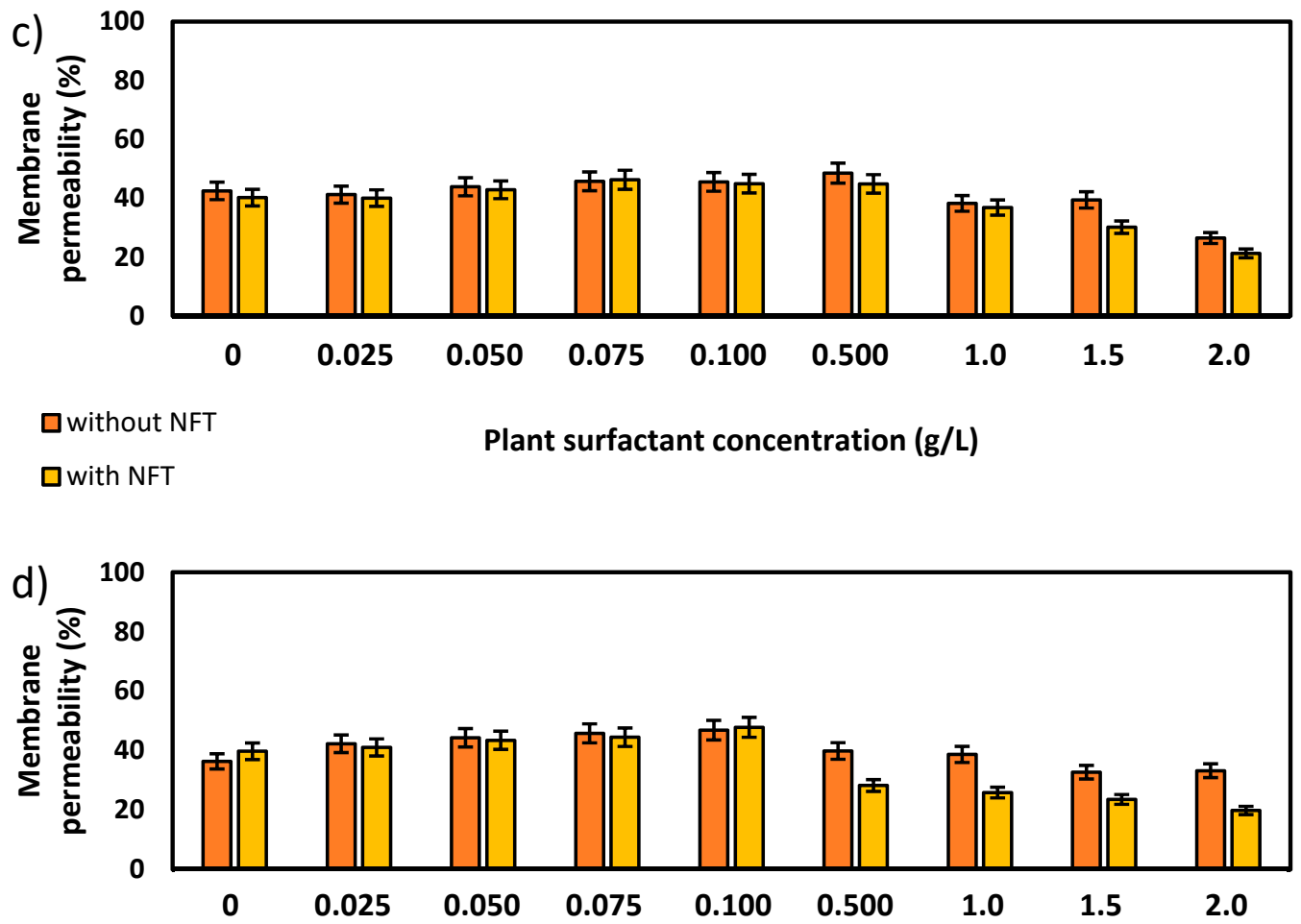

$\square$ without NFT

$\square$ with NFT

\section{Plant surfactant concentration (g/L)}

Figure 4. Total membrane permeability of Pseudomonas sp. MChB strain (a,b) and Achromobacter sp. KW1 (c,d); as measured after $15 \mathrm{~min}(\mathbf{a}, \mathbf{c})$ and $120 \mathrm{~min}(\mathbf{b}, \mathbf{d})$; NFT-nitrofurantoin.

\subsection{Adhesion on Cell Surface}

Additional information about the effects of both, plant extract and NFT, was provided by the studies of adsorption of Congo red dye on the surface bacterial cells (Figure 5). For the first of the tested strains, Pseudomonas sp. MChB, the dye adsorption on the cells surface was at a level of $25 \%$ (after $15 \mathrm{~min}$ ) and reached nearly $40 \%$ after $120 \mathrm{~min}$. When the plant surfactant concentration increased, the adsorption slightly decreased, which was more visible when measurements were conducted after 120 min (Figure 5b). At $2 \mathrm{CMC}$ the Congo red adsorption dropped down to $17 \%$. Moreover, the additional presence of NFT limited the adsorption to $6 \%$. For Achromobacter sp. KW1 the changes in dye adsorption were clearly visible already after $15 \mathrm{~min}$. For the cells exposed to the surfactant only, the decrease from $30 \%$ (in control sample) to $12 \%$ at 2 CMC was observed. However, the impact of NFT exposure on the cell surface is ambiguous for both strains. Nevertheless, it could be concluded that the plant extract was more influential than NFT and the differences between the samples exposed and non-exposed to NFT (but at the same surfactant concentration) did not exceed 5 percent points. Only for Pseudomonas sp. MChB cells at the plant extract concentration of 2 CMC after 120 min a significant difference between the samples without and with NFT was noted. 

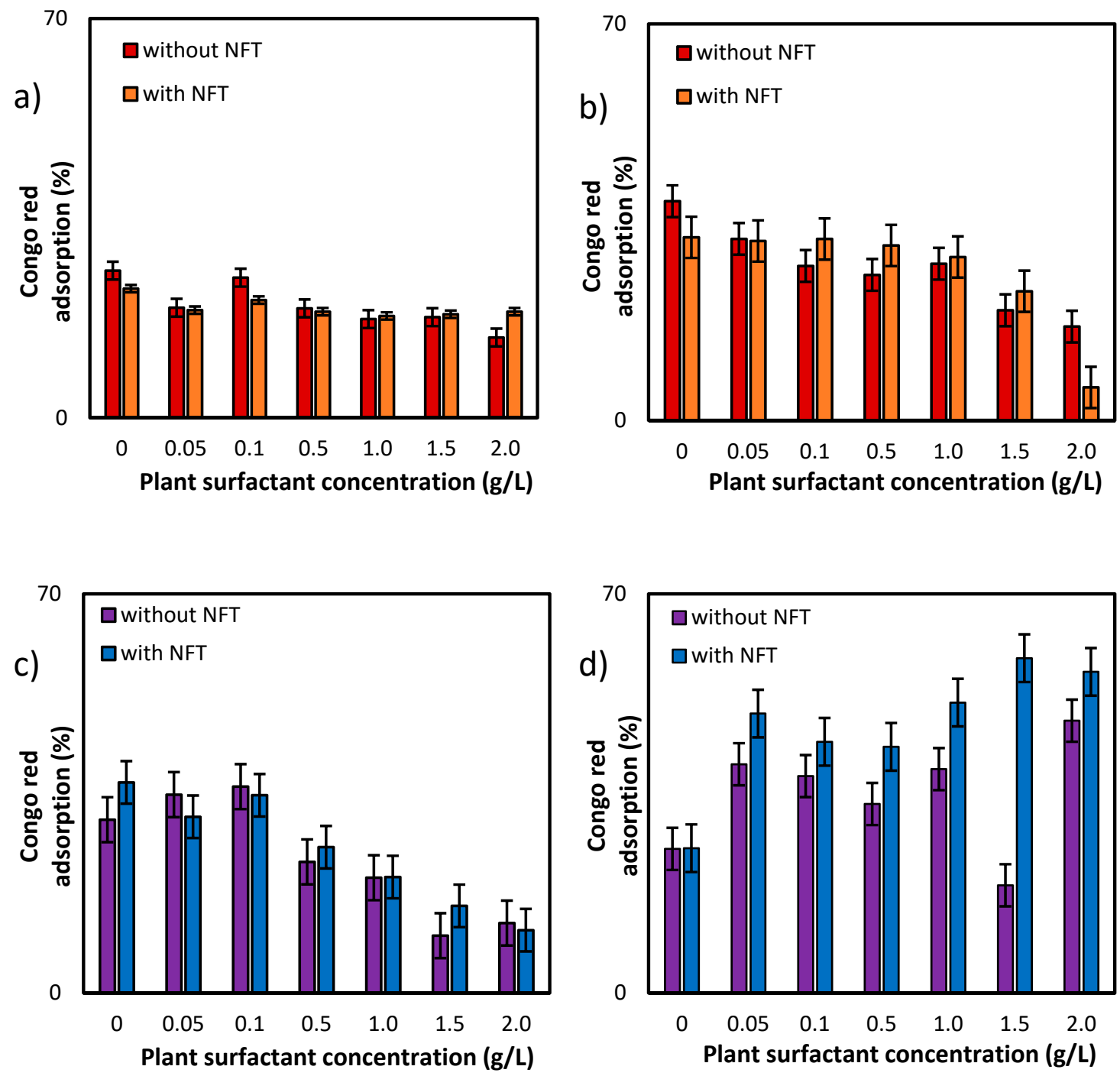

Figure 5. Congo red adsorption on the cell surface of Pseudomonas sp. MChB strain (a,b) and Achromobacter sp. KW1 (c,d); the measurements after $15 \mathrm{~min}(\mathbf{a}, \mathbf{c})$ and $120 \mathrm{~min}(\mathbf{b}, \mathbf{d})$.

\section{Discussion}

The biodegradation process supported by saponins is perceived as a promising solution for the removal of many toxic substances from the environment, including antibiotics. However, literature provides little information on the biodegradation of nitrofuran derivatives by environmental bacteria and on changes in the structure and metabolism of bacterial cells caused by contact with this group of compounds and the extract of saponins. Modifications at the bacterial cell membrane level are also of key importance for determination of the ability of individual strains to degrade nitrofuran derivatives in the presence of saponins. There are many literature reports $[16,17]$ on the interaction of saponins with model cell membranes, however, not in the presence of antibiotics. Korchowiec et al. [18] have shown that saponins interact with model membranes and change the physical state of membranes by perturbing the lipid acyl chain orientation. Moreover, the chemical structure of the natural surfactant containing sugar groups as substituents, weakens their binding to the oppositely charged ions and causes the dissolution of phospholipid membranes in comparison with their chemical counterparts [19]. The important consequence is the surfactants tendency to insert into the membranes rather than dissolving them. There are suggestions [20] that saponin monomers insert into the outer leaflet and induce a rapid increase in the area difference between the two leaflets, which leads to a positive membrane curvature and formation of specific domains whose 
size increases over time. This process is accelerated by the presence of sugar chains, which help develop membrane defects and gradually permeabilize the membrane. However, as far as saponins solutions above CMC are concerned, the saponin micelles can locally increase concentrations of saponins and may be more conducive to the permeabilization process [4]. This is consistent with our results, as we observed an increase in surface area per molecule in the monolayer over time in the presence of saponins extract, regardless of the concentration of surfactant solution. However, the question is what is the effect of the nitrofurantoin presence in the solution on the interactions of the monolayer with saponins.

On the basis of the obtained data, NFT slightly interacts with the phospholipid monolayer leading to a reduction of the area per molecule over time. A similar effect has been reported in the study of Machado and Casei [1], who compared the stability curves of phospholipid DPPS with or without NFT. According to these authors, the process of relaxation in the presence of NFT causes a decrease in the monolayer surface pressures. Consequently, it induces a more compressible state of the monolayer. Moreover, it has been shown that the interaction between phospholipid and NFT can lead to the formation of interfacial aggregates.

However, what is important is that the presence of NFT had a significant impact on the penetration process of the monolayer through the plant surfactant molecules. However, the impact of the NFT addition on the plant extract containing system is ambiguous. In the case of premicellar saponins solution (0.5 CMC), the addition of NFT slowed down the incorporation of surfactant molecules into the phospholipid monolayer. It can be assumed that this effect is caused by the competitive interactions between saponins and NFT, which simultaneously limit the adsorption process of surfactants components on the monolayer.

The opposite effect was found for the micellar solutions of saponins. Above the CMC, the surfactant micelles may also interact with NFT but in a different way. This implicates that the micelles solubilize the antibiotic and enclose it inside them. The entrapment of the NFT inside the micelles may limit the competitive interactions that can be found in the premicellar solution. However, on the other hand, micelles could play a role as transporter of NFT towards the monolayer and release this compound during its diffusion process. Probably, released NFT can also interact with POPE, which leads to the formation of domains with greater area per molecule in comparison with those obtained in the experiments with Saponaria extract only. This effect is especially well visible for the saponins solution of 1.0 CMC. Machado and Casei [1] have shown that NFT can be incorporated in the phospholipids monolayer and form large aggregates. However, it should also be emphasized that the incorporation process may vary depending on the chemical structure of the polar head of each phospholipid. Therefore, for some phospholipids, despite the formation of large aggregates, which would probably disrupt the monolayer, formation of a more stable and ordered monolayer was observed.

Although the permeability of the bacterial cell membrane in the presence of various surfactants has already been analyzed, relatively few studies have been devoted to the effects of saponins, especially those present in the extract of Saponaria officinalis. Smułek et al. [21] have observed that the extract caused an increase in inner membrane permeability of Pseudomonas putida DA1 and Achromobacter sp. SA1 strains. However, Kaczorek et al. [5] have reported that Quillaja saponaria bark saponins and Sapindus mukorossi fruits saponins caused a significant reduction in the inner membrane permeability of Raoultella planticola WS2 and Pseudomonas sp. OS2 cells membranes.

Combined interpretation of the results obtained from the model and biological experiments allows proposing a possible mechanism of interaction of saponins with a phospholipid membrane. On the one hand, the results of model membrane relaxation measurements indicate the saponin penetration of the membrane. On the other hand, the biological test shows a decrease in crystal violet permeation in the membrane and decrease in Congo red adsorption. It can be explained if we assume that saponins were inserted into the membrane but only with their hydrophobic part, while the hydrophilic part of saponins remained on the membrane surface (Figure 6), which limits the access of Congo red to the membrane. This interpretation is consistent with the analysis of Otzen [19], who concluded 
that permeation of the bacteria membrane by saponins occurs gradually and intercalation of the sugar part of the molecule happens relatively slower than the fast intercalation of the hydrophobic hydrocarbon chain.

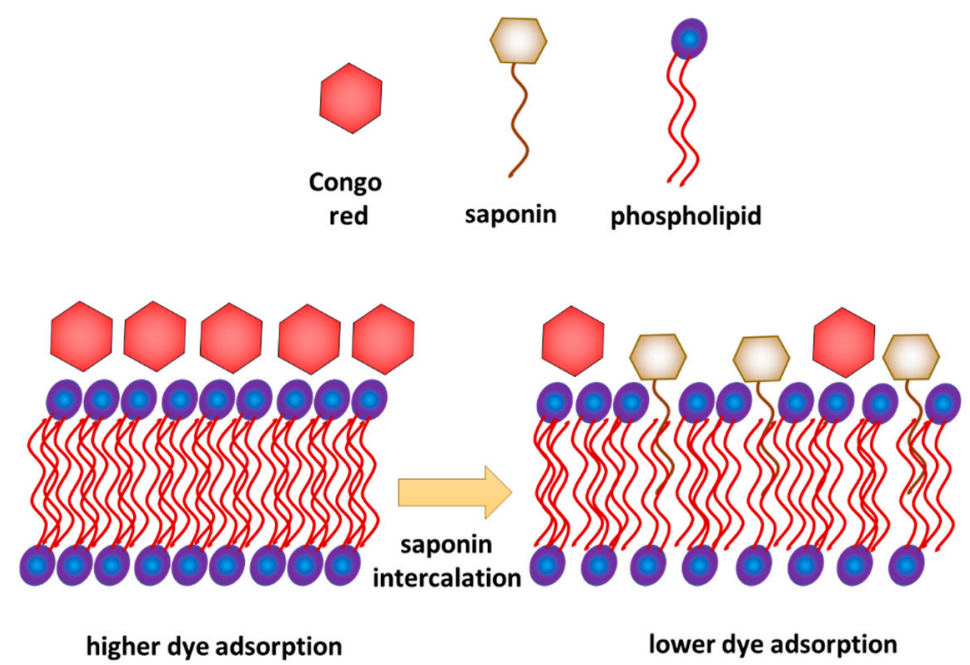

Figure 6. Intercalation of Saponaria officinalis saponins into bacterial phospholipid membrane and its influence on Congo red adsorption-proposition of the mechanism.

It is worth mentioning that for both bacterial strains above the specified concentration of the plant extract, in the presence of nitrofurantoin (NFT) the membrane permeability was higher than in the samples not containing this pharmaceutical. In the case of the Achromobacter strain (though not so visible for the Pseudomonas strain) it is further observed that this difference was more significant after prolonged exposure of the cells to plant extract and NFT (Figure 4). It can be assumed that a stronger intercalation of saponins molecules into the cell membrane (at their higher concentrations) results in a change in the hydrophobicity of the cell surface and, as a result, an increased NFT absorption (contrary to Congo red adsorption-Figure 5). The presence of both hydrophilic saponins groups as well as adsorbed NFT molecules on the cell surface presumably limits cell access to crystal violet particles, whose penetration is analyzed in the used membrane permeability test. At lower concentrations of saponins, their intercalation and, as a result, the modification of the cell surface is not strong enough to affect NFT adsorption, although it is sufficient to change the permeability of the cell membrane to the crystal violet molecules.

The influence of NFT on membrane permeability is a separate issue. While in the model system with POPE the NFT impact is clear, though ambiguous, in the biological system the impact of NFT is practically imperceptible. However, it can be assumed that the model system measurements are more sensitive and the membrane reaction to NFT contact is more noticeable. In the biological system, the cell response is more strongly dominated by activity of plant surfactants.

\section{Materials and Methods}

\subsection{Chemicals}

In the presented studies, the film-forming substance was 2-oleoyl-1-palmitoyl-sn-glycero3-phosphoethanolamine (POPE, 99\%; Sigma-Aldrich, Poznan, Poland). Chloroform of high-purity Uvasol (Merck, Warszawa, Poland) was used to prepare the spreading solutions. Any other chemicals were purchased from Sigma-Aldrich, Poland. For all water solutions, ultrapure water $(18 \mathrm{M} \Omega \cdot \mathrm{cm}$, $71.98 \pm 0.01 \mathrm{mN} / \mathrm{m}$ ) was used. Plant extract from Saponaria officinalis roots (Flos, Mokrsko, Polska) was prepared in the Soxhlet apparatus (Chemland, Stargard, Poland) with methanol as a solvent according to the method described by Smułek et al. [21], and its CMC was evaluated at $1 \mathrm{~g} / \mathrm{L}$. For experiments 
phosphate buffer was prepared (composition in $\mathrm{g} / \mathrm{L}: \mathrm{Na}_{2} \mathrm{HPO}_{4} \cdot 2 \mathrm{H}_{2} \mathrm{O} 7.0, \mathrm{KH}_{2} \mathrm{PO}_{4} 2.8, \mathrm{NaCl} 0.5$ and $\mathrm{NH}_{4} \mathrm{Cl}$ 1.0).

\subsection{Model Membrane Experiments}

After formation of the phospholipid monolayer, the subphase (buffer) could be replaced with a new solution (buffer with saponins, buffer with NFT or buffer with saponins and NFT) by using a peristaltic pump (MINIPULS 3, Gilson, Middleton, WI, USA). A detailed description of the measurement has been provided in our previous paper [22]. The inlet and outlet hoses of pump were mounted to the Langmuir trough on the opposite sides, therefore the surface area of Langmuir through was constant and equal to $225 \mathrm{~cm}^{2}$ (Figure 7).
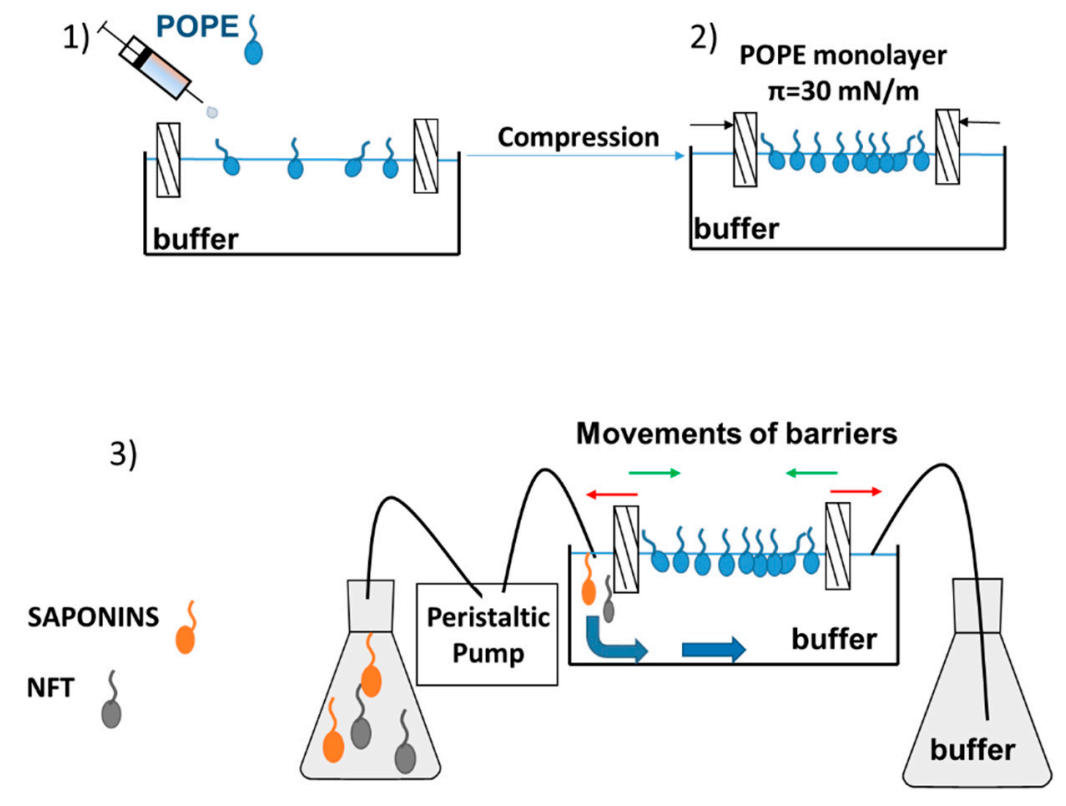

Figure 7. Relaxation measurement using the Langmuir monolayer technique with a dosing pump.

The following procedure was applied in the measurements:

1. Purification of the subphase (by closing the barriers and sucking the contaminations from the buffer surface) and spreading the phospholipids dissolved in a volatile solvent (chloroform),

2. Compression of the monolayer to $\pi=30 \mathrm{mN} / \mathrm{m}$ and waiting $15-20 \mathrm{~min}$ for stabilization of the film and evaporation of chloroform; in the meantime, the new subphase should be prepared and sucked into the pump tubing,

3. Buffer subphase replacement with a new one containing the additional substance/ substances, at a flow rate equal to $13 \mathrm{~mL} / \mathrm{min}$,

4. After the exchange of subphase and setting the pressure of the monolayer between the barriers at $30 \mathrm{mN} / \mathrm{m}$ the Saponaria extract molecules only or with NFT were observed to get adsorbed at the interface.

The relaxation experiments were performed for the pure POPE monolayer and mixed systems: POPE-saponins and POPE-saponins-NFT. The POPE film was initially compressed to a desired surface pressure $30 \mathrm{mN} / \mathrm{m}$ and then the surface area values were recorded over time. To study the POPE-saponins-NFT interactions, the POPE monolayer was compressed to a desired surface pressure and after that the saponins or saponins + NFT solution was pumped underneath the film to the subphase. The observed changes in molecular area were presented as $\mathrm{A} / \mathrm{A}_{0}$, which is the ratio of the actual molecular area in time $t$ to the molecular area at the moment of injection at a constant surface pressure. As a result of the conducted experiments, the relaxation isotherms of monolayer 
were obtained. The temperature of the process $\left(25^{\circ} \mathrm{C}\right)$ was kept constant and controlled during measurements by a Julabo F-12 circulator (Cole-Parmer, Wertheim-Mondfel, Germany).

\subsection{Bacteria Experiments}

Two environmental bacterial strains were used in the experiment: Achromobacter sp. KW1 (NCBI GenBank accession No. KP096516) and Pseudomonas sp. MChB (NCBI GenBank accession No. KU563540). The fatty acids profiles of both strains were characterized by the FAME method [23]. The dominant fatty acids were: palmitic acid (both 30\%), palmitoleic acid (both $25 \%$ ) and vaccenic acid (23\% and 19\% for Achromobacter sp. KW1 and Pseudomonas sp. MChB, respectively).

The bacteria were cultivated for $72 \mathrm{~h}$ with shaking at $120 \mathrm{rpm}$ (KS 4000 ic control, IKA Werke GmbH, Staufen, Germany) in sterilized nutrient broth. Then, after centrifuging at $4000 \times g$ for $10 \mathrm{~min}(3-18 \mathrm{~K}$, Sigma Laborzentrifugen $\mathrm{GmbH}$, Osterode am Harz, Germany) the biomass was washed twice with buffer and finally it was resuspended in the buffer to reach the final bacteria concentration of $1 \times 10^{9} \mathrm{cfu} / \mathrm{mL}$.

Total membrane permeability was tested according to [24] by colorimetric measurements of the uptake of crystal violet solution by microbial cells (CV assay). The cell surface hydrophobicity was analyzed by measuring the adsorption of Congo red dye on the surface of microbial cells (CR assay) [25]. Both measurements were conducted after $15 \mathrm{~min}$ and $120 \mathrm{~min}$ of cell exposure to NFT or/and plant extract at known concentrations.

\subsection{Statistical Analysis}

Unless otherwise noted, all experiments were performed in triplicate, then the mean value and standard deviation for the results series were calculated.

All calculations and graphs were prepared using Microsoft Excel 2013 software.

\section{Conclusions}

Micellar solutions of saponins were found to show strong interactions with the model POPE monolayer as well as the bacterial membranes. When present in concentrations above CMC, saponin molecules incorporate into the POPE monolayer, which could result in a decrease in membrane permeability. Moreover, the adhesion of the indicator, Congo red, to the membrane also decreased at high concentrations of surfactants and in the presence of NFT. On the basis of the obtained results, it was assumed that domains of the saponins could be formed in the POPE monolayer. Such domains embedded in the monolayer structure could change the fluidity of the membrane. Moreover, incorporation of saponins and NFT into the bacterial membrane could lead to a change in the membrane wettability and in consequence change its adhesion properties. In this paper we proposed the most possible mechanism of saponins and NFT interactions with the bacterial membrane.

It is noteworthy that for two different bacterial strains, but of relative similar lipids profile, changes in permeability or adhesion greatly differed, although the same concentrations of saponins and NFT were used. It indicates that other membrane components, found alongside the dominant phospholipids, significantly influence its interactions with plant surfactant or/and antibiotic. For this reason, the next stage of the study will concern the interactions between individual components of bacterial membranes with saponins and NFT. Nevertheless, it can be assumed that the saponins incorporate into the bacteria membrane with the lipid part, but their sugar hydrophilic heads remain outside the membrane, which modifies adsorption properties of the cell surface.

Author Contributions: Conceptualization, W.S., M.R., K.P. and E.K.; methodology, software and validation, M.R. and W.S.; writing_original draft preparation, W.S. and M.R.; writing—review and editing, K.P. and E.K.; visualization, M.R. and W.S.; supervision, K.P. and E.K.; project administration, E.K.; All authors have read and agreed to the published version of the manuscript.

Funding: This research was funded by the National Science Center, Poland, grant number 2017/27/B/NZ9/01603.

Conflicts of Interest: The authors declare no conflict of interest. 


\section{References}

1. Machado, A.C.; Caseli, L. Interaction of nitrofurantoin with lipid langmuir monolayers as cellular membrane models distinguished with tensiometry and infrared spectroscopy. Colloid Surf. B 2020, 188. [CrossRef] [PubMed]

2. McOsker, C.C.; Fitzpatrick, P.M. Nitrofurantoin: Mechanism of action and implications for resistance development in common uropathogens. J Antimicrob. Chemother. 1994, 33, 23-30. [CrossRef]

3. Pacholak, A.; Smułek, W.; Zgoła-Grześkowiak, A.; Kaczorek, E. Nitrofurantoin-microbial degradation and interactions with environmental bacterial strains. Int. J. Environ. Res. Public Health 2019, 16, 1526. [CrossRef] [PubMed]

4. Böttcher, S.; Drusch, S. Saponins-Self-assembly and behavior at aqueous interfaces. Adv. Colloid Interface Sci. 2017, 243, 105-113. [CrossRef] [PubMed]

5. Kaczorek, E.; Smułek, W.; Zdarta, A.; Sawczuk, A.; Zgoła-Grześkowiak, A. Influence of saponins on the biodegradation of halogenated phenols. Ecotoxicol. Environ. Safe 2016, 131, 127-134. [CrossRef] [PubMed]

6. Kobayashi, T.; Kaminaga, H.; Navarro, R.R.; Iimura, Y. Application of aqueous saponin on the remediation of polycyclic aromatic hydrocarbons-contaminated soil. J. Environ. Sci. Health A 2012, 47, 1138-1145. [CrossRef] [PubMed]

7. Shao, B.; Liu, Z.; Zhong, H.; Zeng, G.; Liu, G.; Yu, M.; Liu, Y.; Yang, X.; Li, Z.; Fang, Z.; et al. Effects of rhamnolipids on microorganism characteristics and applications in composting: A review. Microbiol. Res. 2017, 200, 33-44. [CrossRef]

8. Schmidt, S.; Heimesaat, M.M.; Fischer, A.; Bereswill, S.; Melzig, M.F. Saponins increase susceptibility of vancomycin-resistant enterococci to antibiotic compounds. Eur. J. Microbiol. Immunol. 2014, 4, $204-212$. [CrossRef]

9. Wang, Y.; McAllister, T.A.; Newbold, C.J.; Rode, L.M.; Cheeke, P.R.; Cheng, K.-J. Effects of Yucca schidigera extract on fermentation and degradation of steroidal saponins in the rumen simulation technique (RUSITEC). Anim. Feed Sci. Technol. 1998, 74, 143-153. [CrossRef]

10. Phan, M.D.; Shin, K. A Langmuir monolayer: Ideal model membrane to study cell. J. Chem. Biol. Interfaces 2014, 2, 1-5. [CrossRef]

11. Orczyk, M.; Wojciechowski, K.; Brezesinski, G. The influence of steroidal and triterpenoid saponins on monolayer models of the outer leaflets of human erythrocytes, E. coli and S. cerevisiae cell membranes. J. Colloid Interface Sci. 2020, 563, 207-221. [CrossRef] [PubMed]

12. de Groot, C.; Muller-Goymann, C.C. Saponin interactions with model membrane systems-Langmuir monolayer studies, hemolysis and formation of ISCOMs. Planta Med. 2016, 82, 1496-1512. [CrossRef] [PubMed]

13. Brown, R.E.; Brockman, H.L. Using monomolecular films to characterize lipid lateral interactions. Methods Mol. Biol. 2007, 398, 41-58. [PubMed]

14. Nowotarska, S.W.; Nowotarski, K.J.; Friedman, M.; Situ, C. Effect of structure on the interactions between five natural antimicrobial compounds and phospholipids of bacterial cell membrane on model monolayers. Molecules 2014, 19, 7497-7515. [CrossRef]

15. Przykaza, K.; Woźniak, K.; Jurak, M.; Wiącek, A.E.; Mroczka, R. Properties of the Langmuir and Langmuir-Blodgett monolayers of cholesterol-cyclosporine A on water and polymer support. Adsorption 2019, 25, 923-936. [CrossRef]

16. Wojciechowski, K.; Orczyk, M.; Gutberlet, T.; Trapp, M.; Marcinkowski, K.; Kobiela, T.; Geue, T. Unusual penetration of phospholipid mono- and bilayers by Quillaja bark saponin biosurfactant. Biochim. Biophys. Acta Biomembr. 2014, 1838, 1931-1940. [CrossRef]

17. Geisler, R.; Dargel, C.; Hellweg, T. The biosurfactant $\beta$-aescin: A review on the physico-chemical properties and its interaction with lipid model membranes and Langmuir monolayers. Molecules 2020, 25, 117. [CrossRef]

18. Korchowiec, B.; Gorczyca, M.; Wojszko, K.; Janikowska, M.; Henry, M.; Rogalska, E. Impact of two different saponins on the organization of model lipid membranes. Biochim. Biophys. Acta Biomembr. 2015, 1848, 1963-1973. [CrossRef]

19. Otzen, D.E. Biosurfactants and surfactants interacting with membranes and proteins: Same but different? Biochim. Biophys. Acta Biomembr. 2017, 1859, 639-649. [CrossRef] 
20. Lorent, J.; Lins, L.; Domenech, O.; Quetin-Leclercq, J.; Brasseur, R.; Mingeot-Leclercq, M.P. Domain formation and permeabilization induced by the saponin alpha-hederin and its aglycone hederagenin in a cholesterol-containing bilayer. Langmuir 2014, 30, 4556-4569. [CrossRef]

21. Smułek, W.; Zdarta, A.; Pacholak, A.; Zgoła-Grześkowiak, A.; Marczak, Ł.; Jarzębski, M.; Kaczorek, E. Saponaria officinalis L. extract: Surface active properties and impact on environmental bacterial strains. Colloid Surf. B 2017, 150, 209-215. [CrossRef] [PubMed]

22. Krajewska, M.; Dopierała, K.; Prochaska, K. Lipid-protein interactions in Langmuir monolayers under dynamically varied conditions. J. Phys. Chem. B 2020, 124, 302-311. [CrossRef] [PubMed]

23. Kaczorek, E.; Sałek, K.; Guzik, U.; Dudzińska-Bajorek, B.; Olszanowski, A. The impact of long-term contact of Achromobacter sp. 4(2010) with diesel oil-Changes in biodegradation, surface properties and hexadecane monooxygenase activity. Int. Biodeterior. Biodegrad. 2013, 78, 7-16. [CrossRef]

24. Devi, K.P.; Sakthivel, R.; Nisha, S.A.; Suganthy, N.; Pandian, S.K. Eugenol alters the integrity of cell membrane and acts against the nosocomial pathogen Proteus mirabilis. Arch. Pharmacal. 2013, 36, 282-292. [CrossRef] [PubMed]

25. Ambalam, P.; Kondepudi, K.K.; Nilsson, I.; Wadström, T.; Ljungh, Å. Bile stimulates cell surface hydrophobicity, Congo red binding and biofilm formation of Lactobacillus strains. FEMS Microbiol. Lett. 2012, 333, 10-19. [CrossRef] [PubMed]

Sample Availability: Samples of the plant extract used are available from the authors. 